\title{
Clinical Decision Support Systems for Increased Access to Psychological Treatment
}

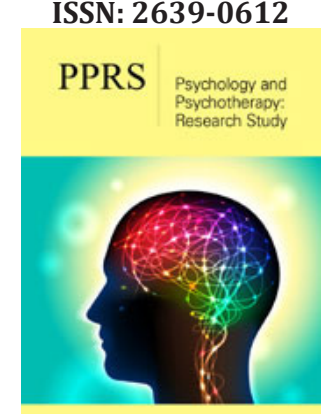

*Corresponding author: Michael Cameron J, University of Southern California, Program in Applied Behavior Analysis, USA

Submission: 眥 August 09, 2019

Published: 眥August 20, 2019

Volume 2 - Issue 4

How to cite this article: Michael Cameron J, Kristen B. Clinical Decision Support Systems for Increased Access to Psychological Treatment. Psychol Psychother Res Stud. 2(4).PPRS.000543.2019.

DOI: 10.31031/PPRS.2019.02.000543

Copyright@ Michael Cameron J, This article is distributed under the terms of the Creative Commons Attribution 4.0 International License, which permits unrestricted use and redistribution provided that the original author and source are credited.

\author{
Michael Cameron J, Ph.D* and Kristen Byra, Ph.D \\ ${ }^{1}$ University of Southern California, Program in Applied Behavior Analysis, USA \\ ${ }^{2}$ The Cedar Group, USA
}

\begin{abstract}
Healthcare professionals are required to make daily decisions about patient care. Moreover, determinations about a specific clinical pathway can have profound impacts on patients' quality of life and long-term outcomes. The decisions made on behalf of a patient often involve practices for which consensus among practitioners is lacking. The divide between clinical science and practice can be bridged by integrating a Clinical Decision Support System (CDSS) into patient care. A CDSS keeps professionals updated with current knowledge and standards of practice in their respective disciplines. This mini review addresses the gap between knowledge and best practice and offers recommendations to bridge the divide accordingly.
\end{abstract}

\section{Psychological Treatment for Pediatric Pain}

Several years ago, I accompanied a colleague and his child to a long-awaited appointment with a psychologist who specialized in pain management. The consultation was for his 17-yearold son who had sustained third-degree burns in an automobile accident. My colleague was seeking individualized, non-drug, interdisciplinary pediatric pain management strategies to address his child's chronic pain. At the conclusion of the consultation, my associate did not receive the nonpharmacological pain treatment protocol expected; rather, he left the appointment with two medication orders the psychologist was authorized to prescribe. The prescriptions were never filled.

\section{Pediatric pain management: Training for psychologists}

According to Law, Palermo, and Walco [1], there are no promulgated pain management guidelines that facilitate the training of pediatric pain psychologists. In consequence, as demonstrated by the opening story, psychologists vary greatly in their ability to assess, prevent, and treat both acute and chronic pain conditions. To address this lack of uniformity in training, there is a need for comprehensive education and instruction for interdisciplinary psychologists who treat children and adults in acute and chronic pain states. In recognition of the importance of pain management in our society, in 2016 researchers from several universities and medical centers collaborated in a survey of six stakeholder groups to determine the perceived need for improved education and training in pain management [2]. The stakeholders surveyed included psychologists, therapists, individuals suffering from chronic pain, pain physicians, primary care physicians, physician assistants, nurse practitioners, and the directors of postgraduate psychology training programs. Based on 1,991 survey responses, these investigators reported that among the professionals, as well as the recipients of pain management treatment, there was "low confidence and low perceived competency to address physical pain." The results of their study supported the need for a national initiative to increase pain management training and competency among healthcare providers [2].

In the absence of comprehensive pain management standards for both practice and care, there is an inevitable risk for indiscriminate treatment, and inadequate and improper pain management. Furthermore, the consequences of poor-quality pain management include the onset of adverse physical and psychological outcomes for patients and their families [3]. There is clearly a need for transformation of the manner in which healthcare providers are trained in the area of pain management, as well as a system for the certification of providers to 
facilitate quality assurance and quality control [2]; however, there is a concurrent need for a knowledge management system that allows healthcare providers to effortlessly and efficiently access critical information regarding pain assessment; pharmacological interventions; non-drug treatment options; and a minimum, standard set of outcome measures most meaningful to patients. The knowledge management needs of healthcare providers can be addressed by way of a Clinical Decision Support System (CDSS).

\section{Clinical Decision Support System and evidence-based guidance}

A CDSS is a decision-making tool (typically technology-enabled) that provides healthcare professionals filtered or targeted clinical information and answers to questions based on patient-specific information. The sequential decision process proposed by Brim [4] comprises five steps:

\section{Identifying the problem}

2. Obtaining necessary information

3. Producing possible solutions

4. Evaluating such solutions, and

5. Selecting a strategy for performance. The results of this sequential process can be applied to the CDSS. The CDSS is intended to provide a step-by-step guide to making decisions tailored to individual patients based on both specified (e.g., pain due to thirddegree burns) and unspecified (e.g., generalized pain) patient problems. When a CDSS is deployed, final individualized decisions are based upon:

a. Patient-specific data and treatment preferences

b. The extant published literature centered on an issue (e.g., pediatric pain management)

c. Evidence-based assessment tools

d. Evidence-based clinical interventions (e.g., Acceptance and Commitment Therapy for pain management)

e. Documented standards of care and standards of practice

f. Ethical principles and codes of conduct

g. Applicable state and federal laws

h. Relevant policies and procedures unique to the organization using the CDSS, and

i. A minimum dataset for outcome measures. The factors that affect clinical decision making include the length of time needed to generate a decision and the amount of information available [5]. The design of the CDSS allows the user to expeditiously access a full scope of information relevant to the current decision, which increases the likelihood of making a decision that maximizes the benefit to the patient.
CDSSs have improved the quality of patient care effectively [6] and are used in medicine to address a wide range of problems. The most effective CDSSs are those that go through a democratized design process with input from content matter experts, end-users, and user experience design (UX-D) and user interface design (UID) professionals. Additionally, serviceable CDSSs offer decisionmaking mobility (i.e., can be used on a tablet), are uncomplicated, unchallenging, and easily used by non-computer-proficient professionals.

\section{Conclusion}

In conclusion, CDSSs allow clinicians to translate all available knowledge and resources into best practice and promote uniformity across a specific discipline. More importantly, the availability of CDSSs enables health providers to access current information centered on treatment (e.g., assessment, intervention, appropriate outcome measures) and facilitate quality of life improvements for patients. Pediatric and adult pain management is just one area that would benefit from the use of CDSSs. Certainly, technology will play a key role in our multimethod approach to reduce morbidity and mortality from opioids; however, we submit that there is a critical need for CDSSs across all disciplines to improve access to evidencebased practices. The body of scientific knowledge that is available to clinicians by way of publications, conference presentations, and media outlets is significant. However, our capacity for information management, knowledge transfer, and knowledge application is limited by the absence of accessible and effective systems. Clinical Decision Support Systems designed collaboratively by content matter experts, user experience design (UX-D) and user interface design (UI-D) professionals will facilitate access to evidence-based psychological treatment and occasion socially significant outcomes.

\section{References}

1. Law EF, Palermo TM, Walco GA (2012) The making of a pediatric pain psychologist: education, training and career trajectories. Pain Manag 2(5): 499-507.

2. Darnall BD, Scheman J, Davin S, Burns JW, Murphy JL, et al. (2016) Pain psychology: A global needs assessment and national call to action. Pain Med 17(2): 250-263.

3. Wells N, Pasero C, McCaffery M (2008) Improving the quality of care through pain assessment and management. In: Hughes RG (Ed.), Patient safety and quality: an evidence-based handbook for nurses. Agency for Healthcare Research and Quality, Rockville, Maryland, USA, pp. 469-497.

4. Hansson SO (2005) Decision theory: A Brief Introduction [college course material]. Department of Philosophy and History of Technology, Royal Institute of Technology (KTH), Sweden.

5. Brodhead M, Cox DJ, Quigley SP (2018) Practical ethics for effective treatment of autism spectrum disorder. Academic Press, Cambridge, Massachusetts, USA.

6. Haynes RB, Wilczynski NL (2010) Computerized clinical decision support system (CCDSS) systematic review team. Effects of computerized clinical decision support systems on practitioner performance and patient outcomes: methods of a decision-maker-researcher partnership systematic review. Implement Sci 5:12.

For possible submissions Click below: 